\title{
29. PETROLOGY OF BASALTS OF HOLES 447A, 449, AND 450, SOUTH PHILIPPINE SEA TRANSECT, DEEP SEA DRILLING PROJECT LEG 59
}

\author{
G. S. Zakariadze, ${ }^{1}$ Janelidze Institute of Geology, Academy of Science of Georgian S.S.R., Tbilisi, U.S.S.R. \\ and \\ L. V. Dmitriev, A. V. Sobolev, and N. M. Suschevskaya, Vernadsky Institute of Geochemistry and \\ Analytical Chemistry, U.S.S.R. Academy of Science, Moscow, U.S.S.R.
}

\begin{abstract}
Basalts studied represent different stages of basement formation of the Philippine Sea plate, including the West Philippine Basin (middle Oligocene-Hole 447A) and the Parece Vela Basin (upper Oligocene to middle MioceneHoles 449 and 450 and Dredge Site 1398, on the seventeenth cruise of the Dmitry Mendeleev). All of these basalts display compositions similar to that of normal mid-ocean ridge (MOR) tholeiites, and despite the age differences, they seem to be differentiation products of an initial tholeiitic melt of essentially the same composition. According to the suggested petrologic scheme, this initial liquid is derived from dry melting of mantle lherzolite at $10 \mathrm{~kb}$ and temperatures about $1270^{\circ} \mathrm{C}$. Basalts of Hole $447 \mathrm{~A}$ were formed after a rapid magma injection to shallow depths and cotectic olivine-plagioclase crystallization at $1 \mathrm{~atm}$, whereas Parece Vela basalts were derived from anchieutectic clinopyroxene-plagioclase-olivine crystallization at intermediate pressures (from $5 \mathrm{~kb}-1 \mathrm{~atm}$ ). In each case, extrusion and crystallization of the magma were preceded by fractionation of excessive olivine from primary tholeiitic liquid. The petrologic and geochemical features of these basalts do not indicate any influence of subduction zone activity. Within tholeitic compositional limits, there is a slight progressive increase of $\mathrm{K}, \mathrm{Ti}, \mathrm{P}$, and $\mathrm{Ba}$ in the basalts and volcanic glasses from the West Philippine Basin to the Parece Vela Basin and thence to the Mariana Trough; this may indicate slightly increasing depths of origin of the primary tholeiitic liquids from west to east along the South Philippine Sea transect.
\end{abstract}

\section{INTRODUCTION}

Petrologic peculiarities provide a key to understanding whether the formation of basalts of marginal sea basins is completely analogous to that of mid-ocean ridge (MOR) tholeiites or whether there are some distinctions related to subduction-zone influence or to other geodynamic factors (Karig, 1971; Packham and Falvey, 1971; Moberly, 1972; Sclater et al., 1976; Toksöz and Bird, 1977; Molnar and Atwater, 1978).

Available data do not present a simple picture. Ridley et al. (1974) and Hawkins $(1976 ; 1977)$ find marginal sea tholeiites and mid-ocean ridge basalts (MORB) completely analogous; other researchers (Hart et al., 1972; Gill, 1976; Tarney et al., 1977; Dietrich et al., 1977) point out distinctions between these two types of basalts. To some extent the petrologic and geochemical features of basement basalts may depend on the genetic type and the stage of evolution of marginal sea basins (Hart et al., 1972; Tarney et al., 1977; Dietrich et al., 1977).

New data obtained from DSDP Legs 58, 59, and 60 shed light on problems of the basement origin of various marginal basins within the Philippine Sea plate. Three major stages of basement formation have been established for the Philippine Sea plate: 50 to 35 m.y. (West Philippine Basin), 30 to 13 m.y. (Parece Vela and Shikoku basins), and 5 to 0 m.y. (Mariana Trough). This episodic evolution is the major geodynamic distinc-

\footnotetext{
${ }^{1}$ Present address: Vernadsky Institute of Geochemistry and Analytical Chemistry, U.S.S.R. Academy of Science, Moscow, U.S.S.R.
}

tion between the Philippine Sea plate and the large oceanic plates (Karig, 1975). Thus it is possible to compare basalts of the Philippine Sea basins with MOR basalts for each stage and also to examine the evolution of basaltic volcanism from stage to stage.

The major objective of this chapter is to present the results of comparative petrologic and geochemical investigation of basement basalts obtained on Leg 59 from the West Philippine Basin (Hole 447A) and the Parece Vela Basin (Holes 449 and 450). These basalts were formed during the first two stages of basin formation of the Philippine Sea plate. Comparison of these basin basalts is of special interest because their geodynamic history may not be completely similar. If the Parece Vela is a good example of an extensional backarc basin, evolution of the West Philippine Basin is much more complex; the several alternative models of its formation are still in dispute (Karig et al., 1973; Karig, 1975; Hilde et al., 1977; and Scott et al., this volume).

\section{SUMMARY OF PETROGRAPHY AND MAJOR PETROCHEMICAL UNITS}

Basements of Holes $447 \mathrm{~A}, 449$, and 450 are composed of aphyric, microphyric, and sparsely phyric basalts showing exceptional petrographic similarity to MOR basalts. Many established petrographic units are composed of well-defined cooling units: (1) single massive lava flows, (2) pillow lava flows, and (3) pillowed massive flows composed of an upper pillow lava underlain by a massive flow. The phenocryst contents are rather limited, ranging from $2 \%$ to $10 \%$; rarely do they amount to $25 \%$. Dominating plagioclase phenocrysts 
$\left(\mathrm{An}_{87-60}\right)$ are represented by slightly resorbed prismatic and tabular crystals $(0.5-3 \mathrm{~mm})$ rarely oscillatory zoned. Subordinate anhedral olivine (0-5\%, 0.5-2 mm) and clinopyroxene $(0-5 \%, 0.5-2.5 \mathrm{~mm})$ are often glomeromorphed with plagioclase. Inner parts of lava pillows and massive flows display ophitic to subophitic textures and are composed of plagioclase $(35-50 \%, 0.4-$ $\left.0.8 \mathrm{~mm}, \mathrm{An}_{67-40}\right)$, clinopyroxene (30-45\%, 0.4-1 mm), olivine $(0-5 \%, 0.1-0.5 \mathrm{~mm})$, titanomagnetite $(0-5 \%$, $0.01-2 \mathrm{~mm})$, and glass (2-10\%). Less crystallized pillow and flow margins enriched with glass reveal textures varying from intersertal to hyalopilitic and variolitic. Plagioclase and olivine phenocrysts in some cases contain small inclusions of brown chromic spinel. Microphenocrysts, microinclusions, and microxenocrysts of chrome spinel also occur in the groundmass. Scattered spherical and irregular vugs $(\sim 5 \%)$ and vesicles $(\sim 3 \%)$ range in size from 0.5 to $5 \mathrm{~mm}$ and from 0.05 to 0.1 $\mathrm{mm}$, respectively; these are partially or completely infilled with carbonates (sometimes with zeolites) rimmed by smectite. The basalts commonly are dissected by veins $(0.5-1 \mathrm{~mm})$ filled mainly by carbonates.

Almost all of the basalt samples studied were more or less subjected to low-temperature secondary alteration. The most unstable constituents are glass (replaced by smectite, zeolite, and, partially, by carbonate) and olivine (replaced by the same mineral assemblage and by serpentine, saponite, and iron hydroxides as well). The major constituents-plagioclase and pyroxene-exhibit only slightly secondary alteration.

Analytic results (Tables 1-6) present clear evidence that all of the basalts are normal MOR tholeiites (Kay et al., 1970; Bougault and Hekinian, 1974; Kay and Hubbard, 1978; Sun et al., in press). Nevertheless, within these compositional limits several obvious petrochemical units are distinguished, based on the composition of volcanic glasses, aphyric rock types, and phyric rock types with a low degree of posteruptive differentiation (Fig. 1-note that unit numbers referred to in this chapter are listed in Column B; these differ from the unit numbers in Column A, which are used in the site report).

Definite compositional variation is related to submarine low-temperature alteration of basalts. Figure 2 illustrates the increase of $\mathrm{K}_{2} \mathrm{O}$ content with progressive oxidation (a factor of 2-3 relative to unaltered samples). The most altered rocks of Hole 447A are represented at the upper (Cores 14-18) and lower (Cores 28 and 35) levels of the basalt section. Altered tholeiites of Sites 449 and 450 display less potassium enrichment. An increase of potassium is accompanied by an increase of $\mathrm{H}_{2} \mathrm{O}^{+}$and $\mathrm{Al}_{2} \mathrm{O}_{3}$ and a decrease of $\mathrm{MgO}$ and $\mathrm{SiO}_{2}$ contents (Table 1, Fig. 3, A-F). On the whole, similar trends are characteristic for alteration of basaltic glasses of Hole 447A (Table 2, Fig. 6).

Let us consider data for the separate sites.

\section{Hole 447A (West Philippine Basin)}

Basaltic basement of Hole 447A (113-296.5 m subbottom) is represented by a continuous section of tholeiitic flows and pillow lavas which are overlain by 28 meters of middle Oligocene volcaniclastic breccias and vitric tuffs. On the basis of petrograph and petrochemical data, the basement section is subdivided into five major petrochemical units (Fig. 1; the unit designations used in this chapter are in Column B of this figure; those in Column $\mathrm{A}$ are used in the site report).

Unit 1 is 28 meters thick (113-141 m sub-bottom) and consists of four flows. The upper three flows are from the top down, respectively, a massive flow $(2 \mathrm{~m})$, pillow lava $(8 \mathrm{~m})$ and a composite flow (pillow lava grading down into a massive flow, $8.5 \mathrm{~m}$ ), all consisting of plagioclase-phyric tholeiite; the lowermost flow is massive aphyric tholeiite $(9.5 \mathrm{~m})$. Plagioclase phenocrysts account for $1 \%$ to $3 \%$, rarely attaining $10 \%$. Low-temperature alteration is rather extensive, mainly at the expense of groundmass olivine and glassy mesostasis, causing a relative decrease in $\mathrm{SiO}_{2}$ and $\mathrm{MgO}$ and increase in $\mathrm{Al}_{2} \mathrm{O}_{3}$ and $\mathrm{K}_{2} \mathrm{O}$ contents (Figs. 2 and 3, A, C, and E).

Unit 2 consists of a composite tholeiitic flow 13.5 meters thick (141-154.5 m sub-bottom; Fig. 1) and is composed of an upper pillow lava $(12.1 \mathrm{~m})$ underlain by a massive flow. Phenocrysts are represented by the uniform assemblage of plagioclase $\left(\mathrm{An}_{72-69}, 3-10 \%\right)$, clinopyroxene (3-6\%), and olivine (1-2\%). The groundmass has a high content of titanomagnetite $(0.5-7 \%)$, which increases total iron and titanium contents of the basalt as compared to the other units in Hole 447A. As in Unit 1, olivine phenocrysts and olivine in the groundmass and glass are considerably altered; the basalts are respectively low in magnesium and silica and enriched in potassium. Because of superimposed low-temperature alteration, the basalts of Units 1 and 2 have an unusual composition that is off the main petrochemical trend of Hole 447A tholeiites (Fig. 3, A, C, and E).

Although the basalts of Units 3 and 5 show some petrographic distinctions, petrochemically they form a common group. Unit 3 (154.5-184.5 m sub-bottom; Fig. 1) consists of three flows: an upper plagioclaseclinopyroxene-olivine microphyric tholeiite $(12.5 \mathrm{~m})$; a middle tholeiite similar to the one above but impoverished in phenocrysts and enriched with glass (up to 80 or $90 \%, 4 \mathrm{~m}$ ); and a lower plagioclase-clinopyroxeneolivine phyric dolerite $(13.5 \mathrm{~m})$. Each consists of an upper pillow lava underlain by a massive flow. In most cases, phenocrysts are represented by the three-mineral assemblage: plagioclase $\left(\mathrm{An}_{82-70}\right)$, clinopyroxene (2$3 \%$ ), and olivine (1\%). Microprobe analysis of some of these phases are given in Tables 3 through 5 .

Unit 5 is composed of six flows with a total thickness of 88 meters. Down-section three composite flows 19.5, 7 , and 5 meters thick, respectively, are underlain by three pillow lava flows, respectively, $6.5,35$, and 14.5 meters in drilled thickness. Petrographically the flows are plagioclase $\left(\mathrm{An}_{85-72}, 2-10 \%\right)$, olivine $(1-5 \%)$, phyric basalts containing small inclusions of chrome spinel $(0.01-0.02 \mathrm{~mm})$ in phenocrysts and in the groundmass. At the base of the section, the basalts contain xenocrysts of spinel and plagioclase associated with small $(5 \mathrm{~mm})$ 
A

B

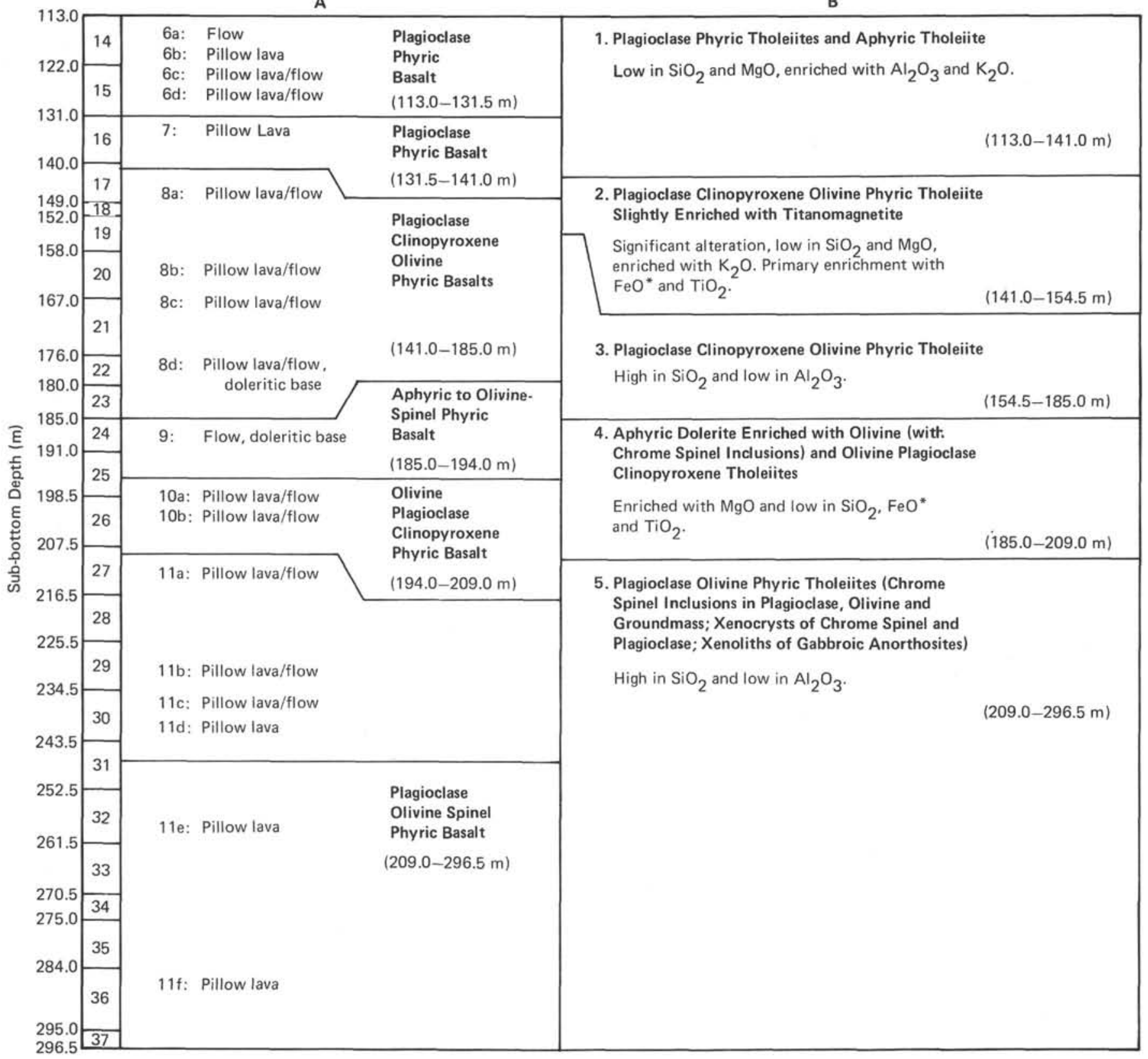

Figure 1. Major petrochemical units of basement section of Hole 447A. (The unit numbers in the A column are those used in the site report; note that they differ from the numbers in the B column, which represents the unit divisions referred to in this chapter.)

xenoliths of gabbroic anorthosites. Despite these petrographic differences, the rocks of Units 3 and 5 petrochemically form an indivisible group that distinctly dominates the basement section of Hole 447A. Basalts of this group are the most siliceous and are relatively low in magnesium and alumina (Fig. 3). The composition of the whole rocks and the glasses of Units 3 and 5 show no significant differences and vary within rather narrow limits, which is evidence for negligible posteruptive fractionation of phenocrysts.

The upper part of Unit 4 is of special interest. It consists of a massive aphyric doleritic flow (185-194.0 m sub-bottom, Fig. 1). The distinctive feature of this unit is marked enrichment in olivine (up to $15 \%$, especially at the base of the flow), containing inclusions of brown chrome spinel $(0.1-0.2 \mathrm{~m})$. The olivine is extensively resorbed to skeletal forms and obviously is the earliest phase that crystallized before plagioclase. Petrochemically the doleritic flow is the most magnesian rock $[\mathrm{MgO} /(\mathrm{MgO}+\mathrm{FeO})=0.52]$, the least siliceous $\left(45.55 \% \mathrm{SiO}_{2}\right)$, the least ferriferous $\left(2.88 \% \mathrm{Fe}_{2} \mathrm{O}_{3}\right.$ and $5.66 \% \mathrm{FeO})$, and the least titaniferous $\left(0.79 \% \mathrm{TiO}_{2}\right)$ in Hole 447A. The alumina content is rather high for a mid-ocean ridge tholeiitic basalt. In addition, the high 


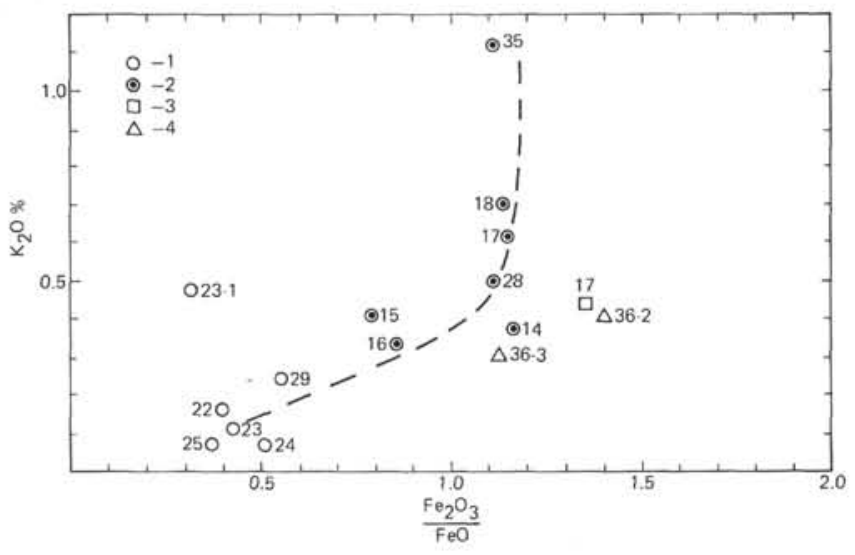

Figure 2. Distribution of $\mathrm{K}_{2} \mathrm{O}$ contents of tholeiites of Holes 447A, 449 , and 450 in relation to the state of alteration of rocks. (In the key 1 and 2 represent fresh and altered tholeiites of Hole 447A, respectively; 3 and 4 are tholeites of Holes 449 and 450, respectively. Numbers refer to cores for respective holes.)

magnesian group includes two other underlying flows of olivine-plagioclase-clinopyroxene tholeiites $(9.5 \mathrm{~m}$ and $5.5 \mathrm{~m}, 194.0-209.0 \mathrm{~m}$ sub-bottom).

\section{Hole 449 (Western part of the Parece Vela Basin)}

Basaltic basement of Site 449 was cored from 111 to 151.5 meters sub-bottom, beneath upper-Oligocene nannofossil ooze. The upper part of the basalt section $(23.5 \mathrm{~m})$ consists of plagioclase-olivine phyric tholeiite $\left(\mathrm{An}_{80-70}, 5-7 \%\right.$; olivine 2-3\%), which is glass-rich between 111 and 124 meters sub-bottom. The underlying 17 meters are composed of a massive plagioclase phyric tholeiitic flow, with plagioclase phenocrysts up to $30 \%$.

\section{Hole 450 (Eastern part of the Parece Vela Basin)}

Only one flow of plagioclase-olivine phyric pillowed tholeiite was encountered and cored (330-340 m subbottom) at Hole 450. Plagioclase phenocrysts in this flow are common $\left(8-16 \%, \mathrm{An}_{80-60}\right.$ core composition, $0.5-6 \mathrm{~mm}$ ) and contain numerous glass inclusions. Olivine phenocrysts are less common (1-2\%) and are completely pseudomorphed by iddingsite, clays, and zeolites. The flow lies beneath a thick volcaniclastic apron of middle-Miocene fine vitric tuffs thermally metamorphosed in contact with the basalt. Seismic-reflection profiles show that these basalts form a rather small prominence (less than $1 \mathrm{~km}$ across) and that true basement is significantly deeper nearby; based on these observations, the basaltic flow of Hole 450 is interpreted as an intrusion that most likely represents a late stage of basement formation (see the Site 450 report, this volume). The considerable petrographic similarity of tholeiites of Sites 450,449 , and 447 favors this assumption. For more detailed petrography, see the site reports.

\section{Other Sites in the Parece Vela Basin}

Data on the composition of Parece Vela Basin basement basalts have also been obtained on DSDP Leg 6 (Site 54, Ridley et al., 1974) and on the seventeenth cruise of the Soviet Dmitry Mendeleev, on which the western slope of the Parece Vela Rift was dredged. (Initial Report of the scientific party 1977, Dietrich et al., 1977). Basalts at Site 54 are microphyric and aphyric plagioclase-olivine tholeiites, petrographically similar to basalts of Holes 449 and 450 (detailed description of these basalts is given by Ridley et al., 1974). At the base

Table 1. Chemistry and normative mineralogy of West Philippine and Parece Vela basin basalts, DSDP Leg 59.

\begin{tabular}{|c|c|c|c|c|c|c|c|c|c|c|c|c|c|c|c|c|}
\hline \multirow{3}{*}{$\begin{array}{c}\text { Hole } \\
\text { Unit } \\
\text { Sample } \\
\text { (intervals in cm) }\end{array}$} & \multicolumn{13}{|c|}{$447 \mathrm{~A}$} & \multirow{3}{*}{$\begin{array}{c}449 \\
17-2 \\
41-51\end{array}$} & \multicolumn{2}{|c|}{450} \\
\hline & \multicolumn{3}{|c|}{1} & \multicolumn{2}{|c|}{2} & \multicolumn{3}{|c|}{3} & \multicolumn{2}{|c|}{4} & \multicolumn{3}{|c|}{5} & & & \\
\hline & $\begin{array}{c}14-2 \\
41-43\end{array}$ & $\begin{array}{l}15-1, \\
24-26\end{array}$ & $\begin{array}{c}16-2 \\
87-89\end{array}$ & $\begin{array}{c}17-3, \\
81-86\end{array}$ & $\begin{array}{l}18-1 \\
71-76\end{array}$ & $\begin{array}{l}22-3 \\
32-40\end{array}$ & $\begin{array}{l}23-1 \\
51-58\end{array}$ & $\begin{array}{l}23-3 \\
73-83\end{array}$ & $\begin{array}{c}24-2 \\
25-39\end{array}$ & ${ }_{104-111}^{25-1}$ & $\begin{array}{l}28-3, \\
52-58\end{array}$ & $\begin{array}{l}29-3, \\
32-40\end{array}$ & $\begin{array}{l}35-2, \\
70-75\end{array}$ & & $\begin{array}{c}36-2 \\
146-150\end{array}$ & $\begin{array}{c}36-3 \\
64-75\end{array}$ \\
\hline $\mathrm{SiO}_{2}$ & 47.32 & 47.34 & 47.80 & 47.82 & 47.96 & 49.46 & 50.11 & 50.30 & 45.55 & 47.43 & 47.86 & 49.60 & 46.98 & 47.52 & 47.01 & 47.94 \\
\hline $\mathrm{TiO}_{2}$ & 1.04 & 1.05 & 0.93 & 1,19 & 1.19 & 1.04 & 1.05 & 1.04 & 0.79 & 0.79 & 0.93 & 1.10 & 0.79 & 1.05 & 0.60 & 1.26 \\
\hline $\mathrm{Al}_{2} \mathrm{O}_{3}$ & 17.31 & 16.96 & 16.28 & 15.11 & 16.09 & 14.71 & 14.79 & 14.64 & 16.37 & 16.86 & 16.01 & 14.70 & 16.98 & 17.00 & 15.98 & 16.16 \\
\hline $\mathrm{Fe}_{2} \mathrm{O}_{3}$ & 5.36 & 4.50 & 4.45 & 6.38 & 6.54 & 2.88 & 2.43 & 3.17 & 2.88 & 2.57 & 5.10 & 3.85 & 5.12 & 5.56 & 5.44 & 4.93 \\
\hline $\mathrm{FeO}$ & 4.61 & 5.71 & 5.21 & 5.56 & 5.71 & 7.10 & 7.82 & 7.28 & 5.66 & 6.68 & 4.58 & 6.89 & 4.63 & 4.09 & 3.86 & 4.40 \\
\hline $\mathrm{MnO}$ & 0.15 & 0.14 & 0.14 & 0.12 & 0.15 & 0.15 & 0.14 & 0.17 & 0.14 & 0.14 & 0.15 & 0.17 & 0.12 & 0.16 & 0.14 & 0.12 \\
\hline $\mathrm{MgO}$ & 5.90 & 5.60 & 6.81 & 6.11 & 6.48 & 7.77 & 7.84 & 7.46 & 8.92 & 9.71 & 7.17 & 8.37 & 5.05 & 6.28 & 5.64 & 6.56 \\
\hline $\mathrm{CaO}$ & 11.98 & 12.13 & 11.98 & 11.32 & 10.79 & 12.21 & 11.46 & 11.55 & 11.92 & 11.40 & 12.03 & 11.88 & 11.90 & 11.18 & 13.01 & 12.17 \\
\hline $\mathrm{Na}_{2} \mathrm{O}$ & 2.50 & 2.70 & 2.66 & 2.39 & 2.51 & 2.29 & 2.16 & 2.22 & 2.13 & 2.22 & 3.24 & 1.74 & 3.79 & 2.42 & 2.58 & 2.67 \\
\hline $\mathrm{K}_{2} \mathrm{O}$ & 0.37 & 0.41 & 0.33 & 0.71 & 0.70 & 0.17 & 0.47 & 0.10 & 0.07 & 0.07 & 0.50 & 0.24 & 1.13 & 0.42 & 0.39 & 0.31 \\
\hline $\mathrm{H}_{2} \mathrm{O}^{-}$ & 1.06 & 1.34 & 1.02 & 1.62 & 1.32 & 0.99 & 0.69 & 0.86 & 1.11 & 0.59 & 1.28 & 0.50 & 1.22 & 2.01 & 1.39 & 1.42 \\
\hline $\mathrm{H}_{2} \mathrm{O}^{+}$ & 1.58 & 1.73 & 2.07 & 1.39 & 1.21 & 0.81 & 0.45 & 0.54 & 0.34 & 1.34 & 0.97 & 1.01 & 2.06 & 2.24 & 2.65 & 1.33 \\
\hline $\mathrm{P}_{2} \mathrm{O}_{5}$ & 0.13 & 0.13 & 0.13 & 0.16 & 0.20 & 0.14 & 0.13 & 0.13 & 0.11 & 0.12 & 0.14 & 0.07 & 0.14 & 0.10 & 0.21 & 0.22 \\
\hline Total & 99.30 & 99.74 & 99.81 & 99.78 & 99.85 & 99.72 & 99.54 & 99.46 & 99.09 & 99.92 & 99.96 & 100.12 & 99.91 & 100.06 & 99.90 & 99.49 \\
\hline Q & - & - & - & 0.30 & 0.40 & 1.18 & 2.64 & 3.61 & - & - & - & 3.48 & - & 0.6 & - & - \\
\hline Or & 2.26 & 2.22 & 2.01 & 3.72 & 4.25 & 1.02 & 2.78 & 0.60 & 0.43 & 0.42 & 03.02 & 1.11 & 6.91 & 2.22 & 2.40 & 1.89 \\
\hline $\mathrm{Ab}$ & 21.87 & 23.59 & 23.26 & 20.89 & 21.81 & 19.78 & 18.35 & 19.15 & 19.04 & 19.16 & 23.67 & 14.68 & 18.42 & 20.97 & 22.76 & 23.34 \\
\hline $\mathrm{Ne}$ & - & - & - & - & - & - & - & - & - & - & 2.37 & - & 7.99 & - & - & - \\
\hline An & 36.13 & 37.21 & 32.58 & 29.67 & 31.42 & 29.99 & 29.76 & 30.28 & 36.88 & 36.58 & 28.32 & 32.26 & 26.90 & 36.44 & 32.21 & 32.25 \\
\hline Wo & 10.21 & 11.38 & 11.68 & 11.39 & 9.28 & 12.91 & 11.38 & 11.39 & 10.37 & 8.48 & 13.28 & 11.50 & 13.88 & 8.59 & 14.06 & 1.97 \\
\hline En $\}$ Di & 6.36 & 7.02 & 7.56 & 6.79 & 5.28 & 8.55 & 7.53 & 7.37 & 7.27 & 5.94 & 8.72 & 7.83 & 8.19 & 5.62 & 9.20 & 7.93 \\
\hline$\left.F_{5}\right)$ & 3.24 & 3.19 & 3.32 & 4.00 & 3.60 & 4.42 & 3.03 & 3.25 & 2.21 & 1.83 & 3.62 & 2.77 & 4.99 & 2.37 & 3.87 & 3.16 \\
\hline En $\} \mathrm{Hy}$ & 6.24 & 5.02 & 4.95 & 8.92 & 8.73 & 11.20 & 12.35 & 11.57 & 6.04 & 9.31 & - & 13.35 & - & 10.74 & 3.07 & 6.30 \\
\hline Fs ${ }^{\prime H y}$ & 3.18 & 2.64 & 2.17 & 5.25 & 5.95 & 4.48 & 4.09 & 5.10 & 1.84 & 2.88 & - & 4.88 & - & 4.61 & 1.29 & 2.52 \\
\hline Fo $\}$ & 1.81 & 0.98 & 3.51 & - & - & - & - & - & 7.11 & 6.59 & 6.69 & - & 3.37 & - & 1.66 & 1.83 \\
\hline $\mathrm{Fa} / 01$ & 1.02 & 0.61 & 1.70 & - & - & - & - & - & 2.38 & 2.24 & 3.06 & - & 2.26 & - & 0.77 & 0.80 \\
\hline II & 2.04 & 1.97 & 1.82 & 2.33 & 2.32 & 2.01 & 2.12 & 2.01 & 1.58 & 1.53 & 1.80 & 2.12 & 1.55 & 2.12 & 3.17 & 2.47 \\
\hline $\mathrm{Mt}$ & 4.71 & 5.56 & 4.60 & 5.64 & 5.75 & 4.78 & 5.56 & 4.99 & 4.21 & 4.43 & 4.53 & 6.01 & 4.62 & 5.56 & 4.41 & 4.41 \\
\hline
\end{tabular}



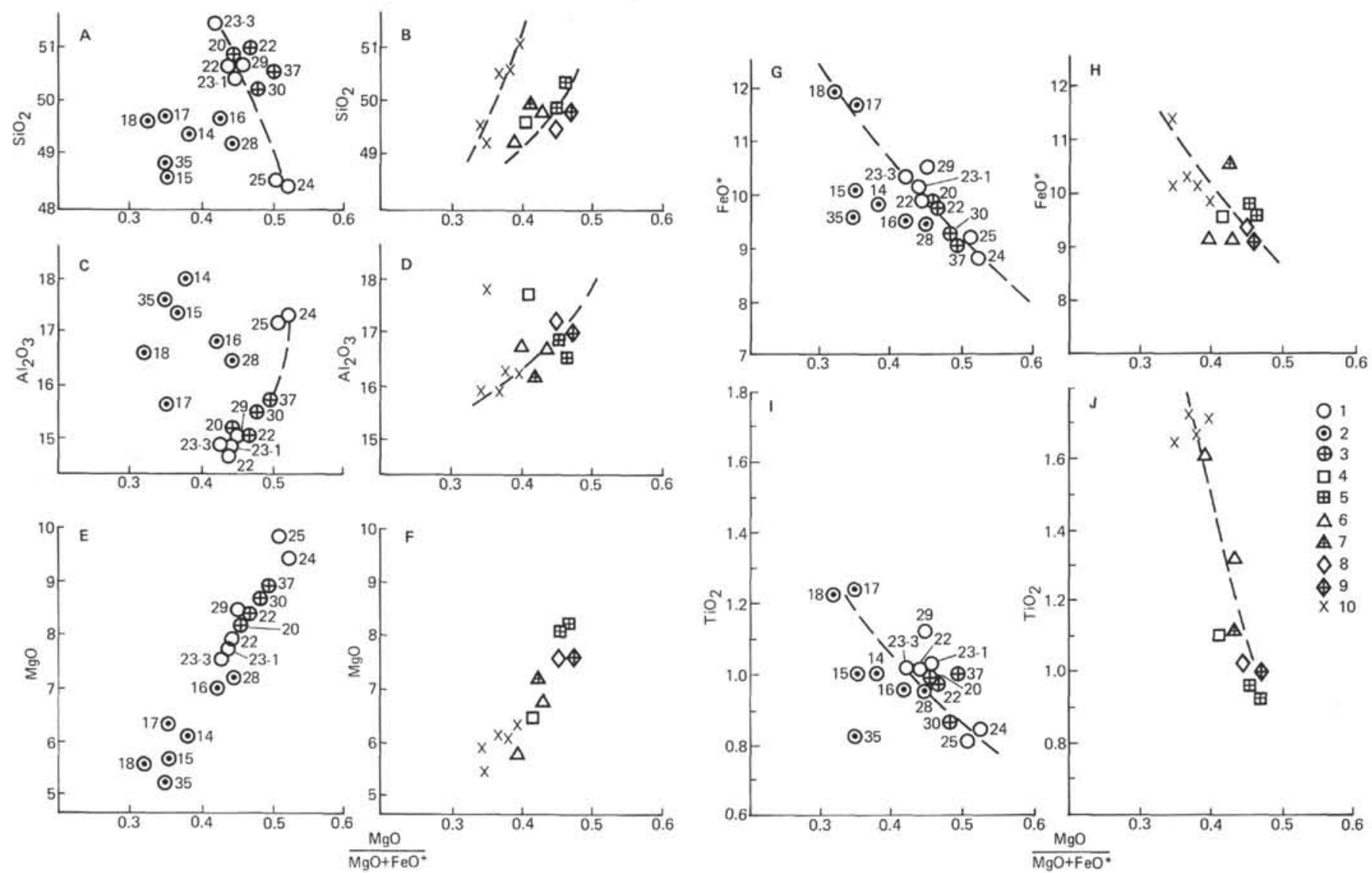

Figure 3. Variation of major-element contents of rocks and glasses of Holes 447A, 449, 450, and 54 in relation to the magnesian number. (Key: 1-3 $=$ Hole $447 \mathrm{~A}-$ fresh whole rocks, altered whole rocks, glasses; $4-5=$ Hole 449 -whole rocks, glasses; $6-7=$ Hole $450-$ whole rocks, glasses; 8-9 = Hole 54-whole rocks, glasses; 10 = Parece Vela Rift tholeiites from Dredge Site 1398, seventeenth cruise of the Dmitry Mendeleev.)

of the western slope of the Parece Vela Rift, three major types of basalts have been dredged (St. $1398,17^{\circ} .07^{\prime} \mathrm{N}$, $\left.139^{\circ} 24^{\prime} \mathrm{E}\right)$ : microphyric and aphyric plagioclase-olivine-clinopyroxene tholeiites, coarsely phyric-plagioclase basalts, and variolitic basalts. Parece Vela Rift microphyric and aphyric tholeiites do not reveal any petrographic distinction from other Parece Vela tholeiites. Plagioclase phenocrysts dominate (5-7\%, $\mathrm{An}_{80-70}$ core composition, $\mathrm{An}_{51}$ marginal zones); olivine and clinopyroxene are present as sparse microphenocrysts. The groundmass is intersertal or hollocrystalline ophitic and consists of coarse plagioclase $\left(40-50 \%, \mathrm{An}_{70-50}\right)$, anhedral clinopyroxene (35-40\%), rare olivine, titanomagnetite $(3-5 \%)$, and partially or completely altered glassy mesostasis. Variolitic basalts represent glass-rich pillow rinds of the tholeiites of the same type. The phyric-plagioclase basalts are extensively altered; plagioclase phenocrysts are often completely pseudomorphed by fine aggregates of smectite and zeolites. The primary composition of these rocks is considerably changed by this alteration.

Analytic data show that there is an apparent difference in the composition of basalts and volcanic glasses of the Parece Vela basement (Tables 1 and 2; Fig. 3), although as a whole these compositions are rather similar to that of MOR basalt. We should emphasize that analyzed glasses were exceptionally fresh, whereas all basalt samples were more or less subjected to low-temperature secondary alteration, mainly at the expense of olivine and glass. Because considerable posteruptive fractionation of phenocrysts within flow units is not observed, noted differences between glasses and crystalline rock in one unit should be explained by lowtemperature alteration processes. Low-temperature alteration is also indicated by the reduced silica and magnesium contents of basalts, similar to altered basalts of Hole 447A. In contrast, the low magnesium, high total iron, high titanium contents, and constant slight oversaturation with silica of Parece Vela Rift tholeiites (Table 1) indicate that these represent the most differentiated rock types of the Parece Vela basement so far obtained.

Trace-element data (Table 6) emphasize the similarity of the West Philippine Basin and the Parece Vela Basin basalts with normal (depleted) MOR tholeiites. Within these limits however, meaningful variations of trace elements have been established. Distribution of trace elements through the section of Hole 447A displays a complex picture, but, as a whole, it is consistent with the major petrochemical units (Fig. 4). (In addition to the Table 6 data, some trace-element data are used from analyses conducted at the University of Birmingham for Leg 59 shipboard scientists.) Parece Vela basalts are slightly enriched with potassium, titanium, and phos- 
Table 2. Microprobe analyses of glasses, DSDP Leg 59.

\begin{tabular}{|c|c|c|c|c|c|c|c|c|c|c|c|c|c|}
\hline \multirow{2}{*}{$\begin{array}{c}\text { Hole } \\
\text { Core-Section, } \\
\text { Interval (in cm) }\end{array}$} & \multicolumn{7}{|c|}{$447 \mathrm{~A}$} & \multicolumn{4}{|c|}{449} & \multicolumn{2}{|c|}{450} \\
\hline & $\begin{array}{l}20-1, \\
9-21\end{array}$ & $\begin{array}{c}22-1 \\
70-76\end{array}$ & \multicolumn{2}{|c|}{$30-5,40-42$} & $\begin{array}{l}37-1, \\
57-60\end{array}$ & \multicolumn{2}{|c|}{$37-1,50-53$} & $15-2$ & \multicolumn{3}{|c|}{$17-1,0-15$} & \multicolumn{2}{|c|}{$36-3,37-42$} \\
\hline $\mathrm{SiO}_{2}$ & 50.67 & 50.75 & 49.67 & 49.61 & $49.46^{\mathrm{a}}$ & 50.16 & 50.27 & 49.72 & 49.85 & 48.95 & 49.59 & $48.82^{b}$ & 49.12 \\
\hline $\mathrm{TiO}_{2}$ & 1.01 & 0.97 & 0.86 & 0.86 & 1.15 & 0.91 & 0.92 & 0.92 & 0.96 & 0.94 & 0.99 & 0.90 & 1.13 \\
\hline $\mathrm{Al}_{2} \mathrm{O}_{3}$ & 15.01 & 14.95 & 15.54 & 15.07 & 16.87 & 15.64 & 16.24 & 16.58 & 16.53 & 16.62 & 16.75 & 15.54 & 16.01 \\
\hline $\mathrm{FeO}$ & 9.83 & 9.76 & 9.27 & 9.17 & 9.95 & 8.94 & 9.46 & 9.40 & 9.52 & 10.00 & 9.50 & 10.41 & 10.47 \\
\hline $\mathrm{MnO}$ & 0.11 & 0.23 & 0.16 & 0.16 & - & 0.18 & 0.18 & 0.18 & 0.16 & 0.18 & 0.18 & 0.18 & 0.10 \\
\hline $\mathrm{MgO}$ & 8.19 & 8.42 & 8.58 & 8.58 & 3.44 & 8.80 & 8.23 & 8.08 & 7.89 & 8.15 & 8.02 & 9.94 & 7.46 \\
\hline $\mathrm{CaO}$ & 12.14 & 12.43 & 13.03 & 12.41 & 12.41 & 12.74 & 11.66 & 11.78 & 11.68 & 11.65 & 12.14 & 11.55 & 11.54 \\
\hline $\mathrm{Na}_{2} \mathrm{O}$ & 2.25 & 2.07 & 1.96 & 2.06 & 2.11 & 1.93 & 2.29 & 2.31 & 2.31 & 2.37 & 2.46 & 2.21 & 2.37 \\
\hline $\begin{array}{l}\mathrm{K}_{2} \mathrm{O} \\
\mathrm{NiO}\end{array}$ & 0.08 & 0.08 & $\begin{array}{l}0.10 \\
0.025\end{array}$ & 0.12 & 0.27 & 0.05 & 0.07 & 0.24 & 0.21 & $\begin{array}{l}0.20 \\
0.022\end{array}$ & 0.24 & $\begin{array}{l}0.20 \\
0.030\end{array}$ & 0.14 \\
\hline Total & 99.29 & 99.66 & 99.19 & 98.04 & 95.64 & 99.35 & 99.32 & 99.21 & 99.11 & 99.08 & 99.87 & 99.87 & 98.34 \\
\hline$\underset{\mathrm{MgO}}{\mathrm{Cr}_{2} \mathrm{O}_{3}}$ & & & & & & & & & & & & 0.10 & \\
\hline$\overline{\mathrm{MgO}+\mathrm{FeO}^{*}}$ & 0.454 & 0.463 & 0.481 & 0.483 & 0.257 & 0.496 & 0.465 & 0.462 & 0.453 & 0.449 & 0.458 & 0.488 & 0.416 \\
\hline Q & - & - & - & - & 2.82 & - & - & - & - & - & - & - & - \\
\hline Or & 0.5 & 0.5 & 0.6 & 0.7 & 1.67 & 0.3 & 0.4 & 1.4 & 1.2 & 1.2 & 1.4 & 1.3 & 0.8 \\
\hline $\begin{array}{l}\mathrm{Ab} \\
\mathrm{Ne}\end{array}$ & 19.0 & 17.5 & 16.6 & $\begin{array}{c}17.4 \\
-\end{array}$ & 18.35 & 16.3 & 19.4 & 19.5 & 19.5 & 20.1 & 20.8 & 18.7 & 20.1 \\
\hline An & 30.6 & 31.6 & 33.3 & 31.5 & 37.55 & 33.9 & 33.8 & 34.2 & 34.1 & 34.1 & 34.0 & 31.8 & 32.6 \\
\hline Wo & 12.4 & 12.7 & 13.1 & 12.5 & 11.26 & 12.2 & 10.0 & 10.1 & 9.9 & 9.9 & 11.0 & 10.6 & 10.3 \\
\hline En $\mathrm{Di}$ & 6.6 & 6.8 & 7.2 & 6.9 & 3.71 & 6.9 & 5.4 & 5.4 & 5.3 & 5.2 & 5.2 & 5.9 & 5.2 \\
\hline Fs & 5.4 & 5.4 & 5.4 & 5.1 & 7.91 & 4.8 & 4.3 & 4.3 & 4.4 & 4.4 & 4.7 & 4.3 & 4.9 \\
\hline & 9.5 & 10.3 & 7.1 & 8.2 & 5.22 & 10.0 & 9.3 & 6.4 & 7.4 & 4.1 & 3.2 & 3.5 & 6.2 \\
\hline Fs $\mathrm{H}$. & 7.7 & 8.2 & 5.3 & 6.1 & 9.23 & 7.0 & 7.4 & 5.1 & 6.1 & 3.4 & 2.6 & 2.5 & 5.8 \\
\hline Fo $\mathrm{Ol}$ & 3.1 & 2.7 & 4.9 & 4.3 & - & 3.5 & 4.0 & 5.8 & 4.9 & 7.7 & 7.7 & 10.8 & 5.1 \\
\hline $\mathrm{Fa} O \mathrm{O}$ & 2.7 & 2.4 & 4.0 & 3.5 & - & 2.7 & 3.5 & 5.1 & 4.50 & 7.2 & 6.8 & 8.5 & 5.3 \\
\hline Il & 1.9 & 1.8 & 1.6 & 1.6 & 2.28 & 1.7 & 1.7 & 1.7 & 1.8 & 1.8 & 1.9 & 1.7 & 2.1 \\
\hline
\end{tabular}

Note: *Indicates total $\mathrm{Fe}$ as $\mathrm{FeO}$.

a Altered glass.

$\mathrm{b}$ Melt inclusion in olivine.

Table 3. Microprobe analysis of plagioclase phenocrysts from DSDP Leg 59 basalts.

\begin{tabular}{|c|c|c|c|c|c|c|c|c|}
\hline \multirow{3}{*}{$\begin{array}{l}\frac{\text { Hole }}{\text { Sample }} \\
\text { (interval in cm) } \\
\mathrm{SiO}_{2}\end{array}$} & \multirow{3}{*}{$\begin{array}{r}20-1 \\
9-21 \\
48.34\end{array}$} & \multicolumn{2}{|c|}{$447 \mathrm{~A}$} & \multicolumn{2}{|c|}{449} & \multirow{2}{*}{\multicolumn{3}{|c|}{$\frac{450}{36-3,37-42}$}} \\
\hline & & \multicolumn{2}{|c|}{$30-5,40-42$} & \multirow{2}{*}{$\begin{array}{c}15-2, \\
50-53 \\
48.21\end{array}$} & \multirow{2}{*}{$\begin{array}{c}\begin{array}{c}17-1, \\
0-5\end{array} \\
49.76\end{array}$} & & & \\
\hline & & 47.50 & 49.86 & & & 46.39 & 49.59 & 51.20 \\
\hline $\mathrm{Al}_{2} \mathrm{O}_{3}$ & & 32.18 & 30.56 & 32.95 & 31.71 & 32.72 & 30.25 & 31.05 \\
\hline $\mathrm{FeO}$ & 0.44 & 0.4 & 0.57 & 0.46 & 0.44 & 0.49 & 0.49 & 0.49 \\
\hline $\mathrm{MgO}$ & 0.32 & 0.37 & 0.37 & 0.24 & 0.23 & 0.30 & - & 0.30 \\
\hline $\mathrm{CaO}$ & 16.34 & 17.3 & 15.38 & 16.76 & 15.50 & 17.62 & 14.67 & 14.42 \\
\hline $\begin{array}{l}\mathrm{Na}_{2} \mathrm{O} \\
\mathrm{K} 3 \mathrm{O}\end{array}$ & $\begin{array}{l}2.0 \\
0.03\end{array}$ & $\begin{array}{l}1.61 \\
0.02\end{array}$ & 2.42 & $\begin{array}{l}2.09 \\
0.03\end{array}$ & 2.28 & 1.33 & 2.88 & 2.75 \\
\hline $\mathrm{K}_{2} \mathrm{O}$ & 0.03 & 0.02 & 0.03 & 0.03 & 0.04 & - & - & 0.02 \\
\hline Total & 99.74 & 99.38 & 99.23 & 100.74 & 100.00 & 98.87 & 97.91 & 100.27 \\
\hline $\mathrm{Si}$ & 2.222 & 2.198 & 2.297 & 2.198 & 2.272 & 2.162 & 132 & 2.323 \\
\hline Al & 1.749 & 1.755 & 1.659 & 771 & & 1.797 & 1.662 & 1.661 \\
\hline $\mathrm{Fe}$ & 0.017 & 0.015 & 0.022 & 0.018 & 0.017 & 0.019 & 0.019 & 0.019 \\
\hline $\mathrm{Mg}$ & 0.022 & 0.026 & 0.026 & 0.016 & 0. & 0.021 & 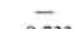 & 0.021 \\
\hline & 0.805 & 0.858 & 0.75 & 0.819 & & 0.879 & 0.733 & 0.701 \\
\hline $\mathrm{Na}$ & 0.178 & 0.144 & 0.21 & 0.185 & 0.202 & 0.121 & 0.261 & 0.242 \\
\hline K & 0.002 & 0.001 & 0.002 & 0.002 & 0.002 & - & - & 0.002 \\
\hline Total & 4.994 & 4.997 & 4.983 & 5.009 & 4.975 & 5.00 & 4.984 & 4.968 \\
\hline An mol $\%$ & 81.9 & 85.6 & 77.63 & 81.6 & 78.96 & 87.91 & 73.73 & 74.17 \\
\hline$\frac{\mathrm{MgO}}{\mathrm{MgO}+\mathrm{FeO}^{*}}$ & 0.58 & 0.52 & 0.61 & 0.66 & 0.66 & 0.62 & - & 0.62 \\
\hline
\end{tabular}

phorus relative to Hole 447A basalts (Figs. 3, D and 8). Possible petrologic aspects of these differences are discussed in following sections.

\section{PETROLOGY}

MOR-type tholeiites originate in the upper mantle under rather narrow limits of temperature and pressure
Table 4. Selective microprobe analysis of olivine phenocrysts from DSDP Leg 59 basalts.

\begin{tabular}{|c|c|c|c|c|c|c|c|c|}
\hline Hole & \multicolumn{5}{|c|}{$447 \mathrm{~A}$} & \multicolumn{3}{|c|}{449} \\
\hline $\begin{array}{l}\text { Sample } \\
\text { (interval in } \mathrm{cm} \text { ) }\end{array}$ & \multicolumn{5}{|c|}{$30-5,40-42$} & \multicolumn{3}{|c|}{$17-1,0-5$} \\
\hline $\mathrm{SiO}_{2}$ & 40.60 & n.d. & n.d. & n.d. & 40.33 & 39.81 & 39.94 & 40.05 \\
\hline $\mathrm{TiO}_{2}$ & n.d. ${ }^{a}$ & n.d. & n.d. & n.d. & n.d. & n.d. & 0.02 & 0.02 \\
\hline $\mathrm{Al}_{2} \overline{\mathrm{O}}_{3}$ & n.d. & n.d. & n.d. & n.d. & n.d. & n.d. & 0.03 & 0.03 \\
\hline $\mathrm{Cr}_{2} \mathrm{O}_{3}$ & n.d. & n.d. & n.d. & n.d. & n.d. & n.d. & 0.04 & 0.03 \\
\hline $\mathrm{FeO}$ & 11.73 & 11.83 & 11.42 & 11.53 & 12.62 & 13.77 & 13.19 & 13.17 \\
\hline $\mathrm{MnO}$ & 0.20 & n.d. & n.d. & n.d. & 0.21 & 0.15 & 0.16 & 0.16 \\
\hline $\mathrm{MgO}$ & 47.90 & 48.02 & 47.94 & 47.84 & 46.45 & 46.26 & 46.22 & 46.34 \\
\hline $\mathrm{CaO}$ & 0.31 & 0.30 & 0.29 & 0.31 & 0.24 & 0.25 & 0.31 & 0.31 \\
\hline $\mathrm{Na}_{2} \mathrm{O}$ & n.d. & n.d. & n.d. & n.d. & n.d. & n.d. & n.d. & n.d. \\
\hline & & & & & & 0.19 & 0.20 & 0.18 \\
\hline Total & 100.0 & & & & 100.02 & 100.39 & 100.09 & 100.20 \\
\hline Si & 0.996 & - & - & - & 1.002 & 0.991 & 0.995 & 0.995 \\
\hline Ti & $-{ }^{b}$ & - & - & - & - & - & 0.000 & 0.000 \\
\hline A] & - & - & - & - & - & - & 0.001 & 0.001 \\
\hline $\mathrm{Cr}$ & - & - & $\overline{1}$ & - & $\overline{0}$ & - & 0.001 & 0.001 \\
\hline $\mathrm{Fe}$ & 0.240 & 0.121 & 0.117 & 0.119 & 0.262 & 0.287 & 0.275 & 0.274 \\
\hline $\mathrm{Mn}$ & 0.04 & & & & 0.004 & 0.003 & 0.003 & 0.003 \\
\hline $\mathrm{Mg}$ & 1.752 & 0.875 & 0.879 & 0.877 & 1.720 & 1.718 & 1.717 & 1.717 \\
\hline $\mathrm{Ca}$ & 0.008 & 0.004 & 0.004 & 0.004 & 0.006 & 0.007 & 0.008 & 0.008 \\
\hline $\mathrm{Na}$ & - & - & - & - & 0.05 & 0.04 & 0.04 & - \\
\hline $\mathrm{Ni}$ & 0.004 & - & - & - & 0.005 & 0.004 & 0.004 & 0.004 \\
\hline Total & 3.004 & - & - & - & 2.999 & 3.010 & 3.004 & 3.003 \\
\hline $\mathrm{Fe}^{+2}$ & \multirow{2}{*}{0.120} & \multirow{2}{*}{0.121} & \multirow{2}{*}{0.118} & \multirow{2}{*}{0.119} & \multirow{2}{*}{0.132} & \multirow{2}{*}{0.143} & \multirow{2}{*}{0.138} & \multirow{2}{*}{0.138} \\
\hline $\mathrm{Fe}^{+2}+\mathrm{MgO}$ & & & & & & & & \\
\hline
\end{tabular}

a Not determined.
No data.

that are sustained during the entire formation of Oceanic Layer 2 (Kushiro, 1973; Fujii and Kushiro, 1976-1977; Bender et al., 1978). The most detailed model of tholeiitic volcanism is based on statistical com- 
Table 5. Selective microprobe analysis of clinopyroxene phenocrysts DSDP Leg 59 basalts.

\begin{tabular}{|c|c|c|c|}
\hline Hole & $447 \mathrm{~A}$ & \multicolumn{2}{|c|}{450} \\
\hline $\begin{array}{c}\text { Sample } \\
\text { (intervals in } \mathrm{cm} \text { ) }\end{array}$ & $20-1,9-21$ & \multicolumn{2}{|c|}{$36-3,37-42$} \\
\hline $\mathrm{SiO}_{2}$ & 52.18 & 49.68 & n.d. ${ }^{\mathrm{a}}$ \\
\hline $\mathrm{TiO}_{2}$ & 0.40 & 0.70 & n.d. \\
\hline $\mathrm{Al}_{2} \mathrm{O}_{3}$ & 2.66 & 4.76 & n.d. \\
\hline $\mathrm{Cr}_{2} \mathrm{O}_{3}$ & 0.55 & 0.83 & n.d. \\
\hline $\mathrm{FeO}$ & 5.88 & 6.00 & 6.37 \\
\hline $\mathrm{MnO}$ & 0.12 & 0.11 & n.d. \\
\hline $\mathrm{MgO}$ & 17.18 & 17.46 & 17.33 \\
\hline $\mathrm{CaO}$ & 19.42 & 19.90 & 18.05 \\
\hline $\mathrm{Na}_{2} \mathrm{O}$ & 0.26 & 0.31 & \\
\hline Total & 98.65 & 99.74 & \\
\hline $\mathrm{Si}$ & 1.932 & 1.833 & $-^{b}$ \\
\hline $\mathrm{Ti}$ & 0.011 & 0.019 & - \\
\hline $\mathrm{Al}$ & 0.116 & 0.207 & - \\
\hline $\mathrm{Cr}$ & 0.016 & 0.024 & - \\
\hline $\mathrm{Fe}$ & 0.182 & 0.185 & 0.106 \\
\hline $\mathrm{Mn}$ & 0.004 & 0.004 & - \\
\hline Mg & 0.949 & 0.961 & 0.512 \\
\hline $\mathrm{Ca}$ & 0.771 & 0.787 & 0.383 \\
\hline $\mathrm{Na}$ & 0.009 & 0.022 & - \\
\hline Total & 4.000 & 4.043 & - \\
\hline $\mathrm{Fe}^{+2}$ & \multirow[b]{2}{*}{0.161} & \multirow[b]{2}{*}{0.161} & \multirow[b]{2}{*}{0.171} \\
\hline$\overline{\mathrm{Fe}^{+2}+\mathrm{Mg}}$ & & & \\
\hline
\end{tabular}

positional data of basaltic lavas and volcanic glasses of the Atlantic Ocean floor and is confirmed by petrologic experiment (Kushiro, 1973; Fujii and Kushiro, 19761977; Bender et al., 1978; Dmitriev et al., 1978). This model is used here for comparison with the petrologic features of the Philippine Sea basement tholeiites.

According to the model, primary tholeiitic melts of the Atlantic are formed in lherzolitic upper mantle under dry conditions from spinel-bearing to plagioclasebearing depths facies. Depths of the origin of primary melts and compositional variation of their differentia- tion products are related to the tectonic regime of evolution of different segments of mid-ocean ridges. Owing to the differences of this regime, melts of the central Atlantic (between the latitudes $25^{\circ} \mathrm{S}$ and $36^{\circ} \mathrm{N}$ ) originate at depths of 15 to $25 \mathrm{~km}$ and those of the northern and southern Atlantic at depths of 30 to $40 \mathrm{~km}$. Apparently for the same reasons, tholeiites of the central Atlantic are less differentiated than tholeiites of the northern and the southern Atlantic (Kushiro, 1973; Fujii and Kushiro, 1976-1977; Bender et al., 1978; Dmitriev et al., 1978; 1979).

Figure 5 is an $\mathrm{Al}_{2} \mathrm{O}_{3}-\mathrm{TiO}_{2}$ diagram that correlates the primary tholeiitic melts and compositional limits of the Atlantic lavas with the composition of Philippine Sea tholeiites (Tables 1 and 2). The figure shows that crystal fractionation of the Philippine Sea basaltic magmas is rather restricted, because rocks with titanium content less than $0.7 \%$ and rocks sharply enriched or impoverished in alumina are completely absent. In contrast, the variation of titanium content is considerable. The lowest concentrations of titanium are in fresh basalts (Unit 4, Fig. 1) and glasses of the lower part of the basement section of Hole 447A, as well as in fresh glasses of Holes 449 and 54. These compositions fall within relatively narrow fields of the cotectic and anchieutectic compositions of Atlantic tholeiites that originated at pressures near $10 \mathrm{~kb}$ and then cooled at low pressures (Dmitriev et al., 1979). Slightly raised titanium content is characteristic of altered basalts of Hole 447A (Unit 2) of basalts from Holes 449,450 , and 54 , and of Parece Vela Rift basalts. Relatively high titanium content is established as well in altered glass of Hole 447A and glass samples of Hole 450 (Table 2).

Figure 6 shows the composition of the same whole rocks and glasses for the olivine-diopside-plagioclase system applied earlier to the model of tholeiitic volcanism of the Atlantic (Dmitriev et al., 1978, 1979). Crosses denote the evolutionary path of tholeiitic liquid formed at $15.5 \mathrm{~kb}$ and then crystallized at $1 \mathrm{~atm}$ within temperature limits of $1205^{\circ}$ to $1270^{\circ}$ (Bender et al., 1978). The figure illustrates good coincidence of compositional variation of whole rocks and glasses of the middle and

Table 6. Trace-element abundances of basalts from Holes 447A, 449, and 450 (ppm).

\begin{tabular}{|c|c|c|c|c|c|c|c|c|c|c|c|c|c|c|c|c|}
\hline Hole & & & & & & & $47 \mathrm{~A}$ & & & & & & 449 & & 45 & \\
\hline $\begin{array}{l}\text { Core-Section, } \\
\text { Interval (in cm) }\end{array}$ & $\begin{array}{l}14-2 \\
41-43\end{array}$ & $\begin{array}{l}15-1 \\
24-26\end{array}$ & $\begin{array}{l}16-2 \\
87-89\end{array}$ & $\begin{array}{l}17-3 \\
81-86\end{array}$ & $\begin{array}{l}\text { 18-1, } \\
71-70\end{array}$ & $\begin{array}{l}22-3 \\
32-40\end{array}$ & $\begin{array}{l}23-1 \\
51-58\end{array}$ & $\begin{array}{l}23-3 \\
73-83\end{array}$ & $\begin{array}{l}24-2 \\
25-39\end{array}$ & $\begin{array}{l}25-1, \\
104-111\end{array}$ & $\begin{array}{l}28-3 \\
52-58\end{array}$ & $\begin{array}{c}35-2 \\
70-75\end{array}$ & $15-2$ & $\begin{array}{l}17-2 \\
41-51\end{array}$ & $\begin{array}{c}36-2 \\
146-150\end{array}$ & $\begin{array}{l}36-3, \\
64-75\end{array}$ \\
\hline $\mathrm{Rb}$ & 6.8 & 4.9 & 4. & 13.5 & 12 & 5 & 0.9 & 1.0 & 0.3 & 0.4 & 4.5 & 14 & 4.5 & 6 & 5.3 & 5 \\
\hline $\mathrm{Ba}$ & 31 & 27 & 30 & 25 & 29 & 20 & 25 & 83 & 26 & 25 & 27 & n.d. ${ }^{a}$ & n.d. & 43 & 41 & 38 \\
\hline $\mathrm{Sr}$ & 120 & 140 & 130 & 91 & 93 & 60 & 71 & 65 & 110 & 130 & 69 & 110 & n.d. & 130 & 120 & 130 \\
\hline Y & 22 & 29 & 29 & 28 & 20 & 33 & 30 & 31 & 23 & 23 & 32 & 34 & n.d. & 22 & 24 & 23 \\
\hline $\mathrm{Cr}$ & 379 & n.d. & 330 & 191 & 175 & 256 & 252 & 250 & 275 & 345 & 452 & 410 & n.d. & 340 & 325 & 333 \\
\hline Co & 49 & 46 & 52 & 52 & 50 & 49 & 47 & 45 & 54 & 60 & 50 & 37 & 36 & 36 & 52 & 52 \\
\hline $\mathrm{Ni}$ & 101 & 110 & 131 & 72 & 64 & 133 & 130 & 150 & 184 & 231 & 128 & 125 & 149 & 176 & 92 & 116 \\
\hline $\mathrm{Cu}$ & 116 & 129 & 116 & 136 & 66 & 109 & 134 & 114 & 120 & 113 & 164 & 85 & 102 & 108 & 64 & 83 \\
\hline $\mathrm{Zn}$ & 98.5 & 100 & 103 & 110 & 115 & 88 & 96 & 90 & 69 & 81 & 105 & 76 & 103 & 89 & 89 & 78 \\
\hline Mn & 1550 & 1720 & 1350 & 1550 & 1570 & 1480 & 1680 & 1610 & 1490 & 1280 & 1370 & 1020 & 1450 & 1240 & 1590 & 1470 \\
\hline P & 572 & 572 & 572 & 704 & 880 & 616 & 572 & 572 & 484 & 528 & 616 & 616 & n.d. & 1056 & 924 & 968 \\
\hline $\mathrm{Ti}$ & 6240 & 6300 & 5580 & 7140 & 7140 & 6240 & 6300 & 6240 & 4740 & 4740 & 5580 & 4740 & 6600 & 6300 & 9600 & 7560 \\
\hline V & 248 & 230 & 233 & 250 & 240 & 308 & 320 & 264 & 170 & 250 & 267 & 261 & 304 & 250 & 320 & 210 \\
\hline
\end{tabular}

a Not determined. 


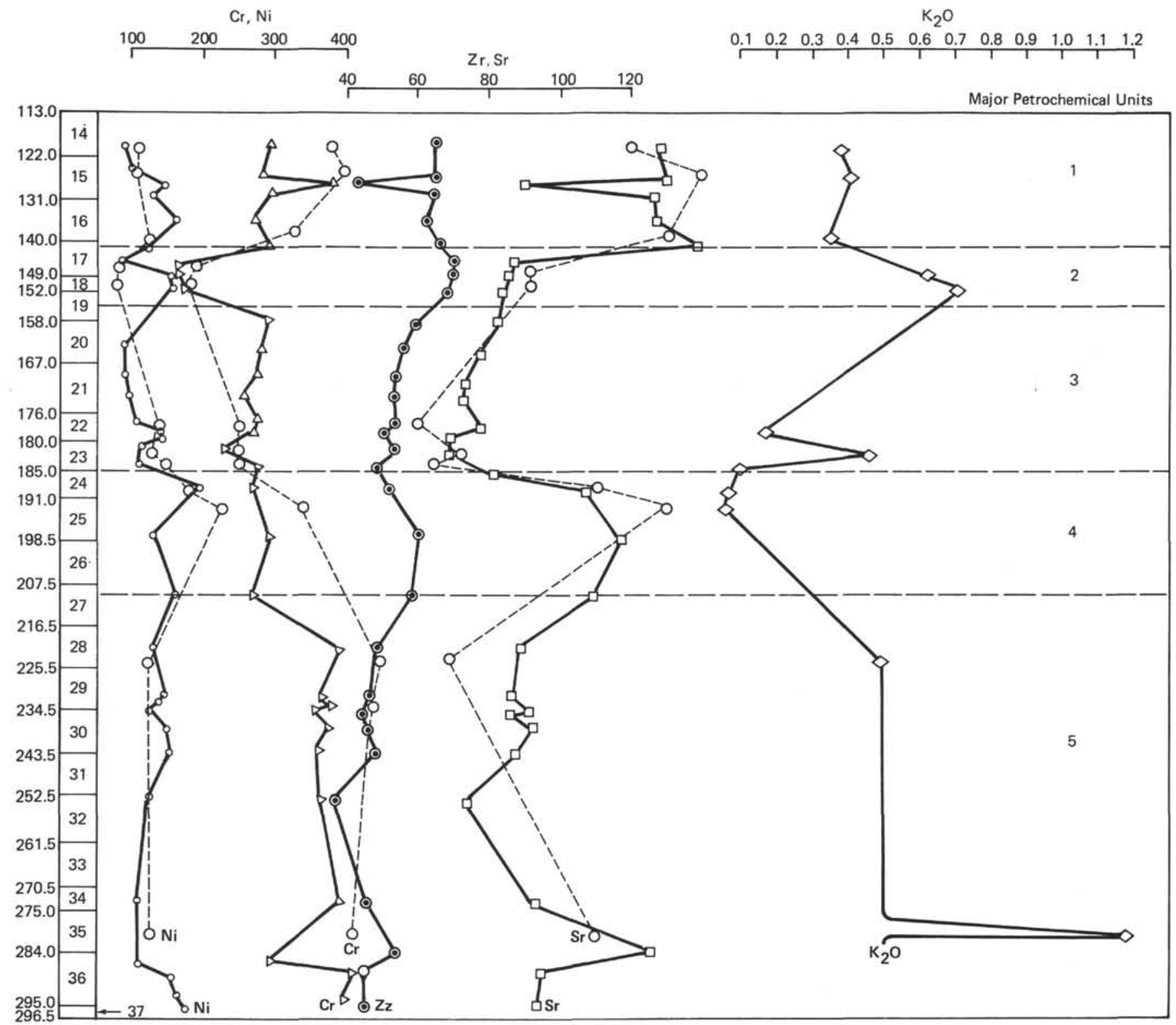

Figure 4. Variation of some trace-element contents along the basement section of Hole 447A. (Solid lines depict analytical data on Leg 59 basalts obtained at the University of Birmingham; dashed lines represent data presented in Table 6.)

lower part of the basement section at Hole 447A (with the variation curve denoted by crosses), except for the lower horizontal part of this curve. This part of the curve represents the initial stage of liquid evolution related to olivine fractionation from the primary melt, which is in equilibrium with mantle lherzolite at $10.5 \mathrm{~kb}$ and then is rapidly uplifted to low pressures without considerable decrease of temperature. Matching experimental data with the major compositional trend of basalts and volcanic glasses of Hole 447A and the established distribution of titanium and alumina most likely indicates that the origin of Site 447 basalts is related to the differentiation of primary tholeiitic liquid, representing partial melt of the upper mantle lherzolite at 10 $\mathrm{kb}$, which then crystallized at shallow depths along the cotectic. In this case, the composition of the volcanic glasses should be close to the composition of low-pres- sure anchieutectic; and the composition of Mg-rich aphyric rocks of Unit 5 should be close to the composition of the melt that most nearly approached that of primary liquid. The differences of the last two are defined by crystallization of olivine at pressure limits from $10.5 \mathrm{~kb}$ to $5 \mathrm{~kb}$, corresponding to olivine crystallization field expansion (Bender et al., 1978). This suggested complex differentiation trend is shown on Figure 6 as Arrow I. The horizontal part of the arrow shows the differentiation path related to crystallization of excessive olivine, whereas the vertical part, in line with the normative clinopyroxene apex, is the path of cotectic crystallization of olivine and plagioclase at low pressure.

Basalts of Unit 4, with the closest to primary-melt composition display, have, as expected, low $\mathrm{K}, \mathrm{La}, \mathrm{Ce}$, $\mathrm{Nd}$, and $\mathrm{Ti}$ contents and high $\mathrm{Ni}$ content. Relative $\mathrm{Sr}$ enrichment of Unit 4 basalts (Fig. 4) is consistent with 


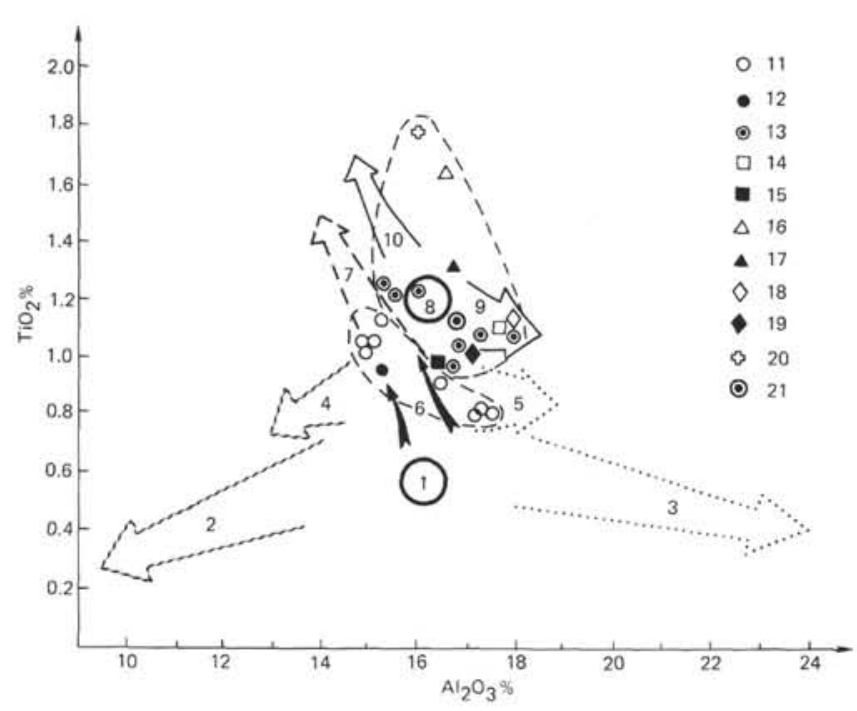

Figure 5. Correlation of $\mathrm{Al}_{2} \mathrm{O}_{3}$ and $\mathrm{TiO}_{2}$ contents of Atlantic tholeiites (Dmitriev et al., 1979) and tholeiites of Philippine Sea basement. (Key: 1 = Primary melt of Atlantic tholeiites originated at $10 \mathrm{~kb} ; 2=$ porphyritic basalts with cumulose olivine; $3=$ porphyritic basalts with cumulose plagioclase; $4=$ glomerophyric basalts $(\mathrm{Ol}-\mathrm{Pl}) ; 5=$ glomerophyric basalts $(\mathrm{Pl}-\mathrm{Ol}-\mathrm{Cpx}) ; 6=$ field of cotectic crystallization; $7=$ field of anchieutectic crystallization; 8 = primary melt of Atlantic tholeiites, originated at 3-5 kb; $9=$ sparsely phyric basalts ( $\mathrm{Pl}-\mathrm{Ol}-\mathrm{Cpx}) ; 10=$ field of anchieutectic crystallization; $11=$ basalts of the lower part of the basement section of Hole 447A; 12 = glasses from the lower part of the basement section of Hole 447A; 13 = basalts of the upper part of the basement section of Hole 447A; $14=$ basalts of Hole 449; 15 $=$ glasses of Hole $449 ; 16=$ basalts of Hole $450 ; 17=$ glasses of Hole $450 ; 18=$ basalts of Hole $54 ; 19=$ glasses of Hole $54 ; 20=$ basalts of Dredge Site 1398 (from the seventeenth cruise of the Dmitry Mendeleev; and 21 = altered glasses of Hole 447A.)

the early fractionation of olivine in the first stage of differentiation and with the cotectic crystallization of olivine and plagioclase that followed. The relatively low chromium content of Unit 4 basalts is possibly the result of an earlier stage of irregular fractionation of spinel inclusions with excessive olivine. It should be emphasized, however, that low chromium content and zirconium enrichment of magnesium-rich and potassium-poor basalts of Unit 4 (Fig. 4) is still to be explained.

The compositions of glasses of Holes 449,450 , and 54 are distributed along a line that branches from the cotectic part of Trend I toward increase of normative plagioclase content (Fig. 6, Arrow II). $\mathrm{Al}_{2} \mathrm{O}_{3}$ and $\mathrm{Na}_{2} \mathrm{O}$ are increased and $\mathrm{CaO}$ is decreased in this direction. Variation of other major elements is insignificant, although compared to that of Trend I of Hole 447A, glasses of the Parece Vela Basin are enriched with Ti, K, and total $\mathrm{Fe}$ and are lower in $\mathrm{Mg}$. In this respect, glasses lying at the end of Arrow II (Fig. 6) are apparently related to the final low-temperature stages of differentiation. Compositional variation along Trend II is possibly caused by the anchieutectic crystallization $(\mathrm{Ol}+$ $\mathrm{Pl}+\mathrm{Cpx})$ of tholeiitic melt. The position of the transitional point from cotectic to anchieutectic crystallization cannot be precisely defined, but the shift of this point toward enrichment of glasses with normative clinopyroxene should be coincident with decrease of

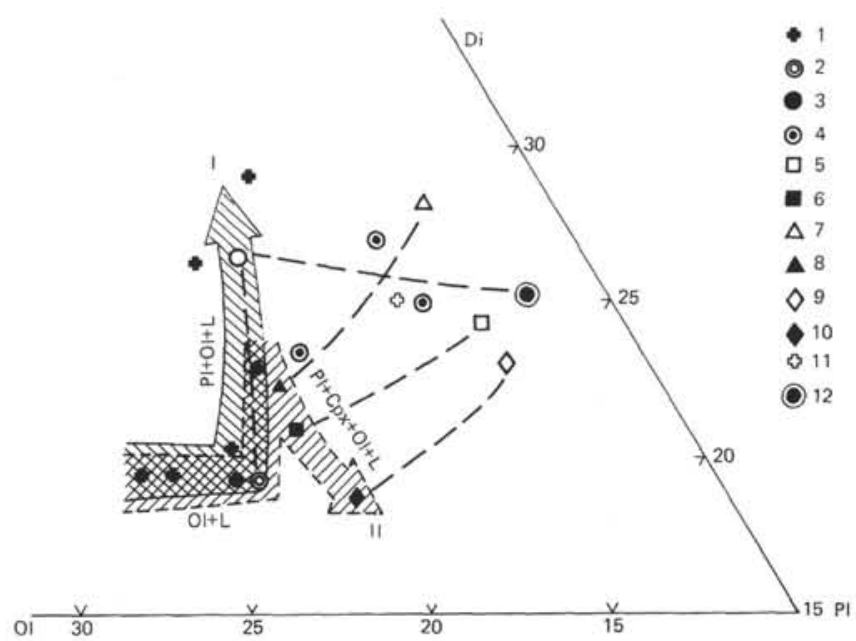

Figure 6. Ol-Pl-Di diagram for compositions of basalts and glasses of Philippine Sea floor compared to experimental data (Bender et al., 1978). (The key shows 1 = composition of the liquid phase of tholeiites, originated at $10.5 \mathrm{~kb}$ and crystallized at $1 \mathrm{~atm}$ (Bender et al., 1978); 2 = average composition of the most magnesian basalts of the Hole 447A (Unit 4); 3 = average composition of volcanic glasses of Hole 447A; 4 = basalts of the upper part of the basement section of Hole 447A; 5 = plagioclase phyric basalts of Hole $449 ; 6=$ average composition of glasses of Hole $449 ; 7=$

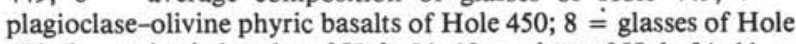
$450 ; 9=$ aphyric basalts of Hole $54 ; 10=$ glass of Hole $54 ; 11=$ plagioclase-olivine-clinopyroxene phyric basalts of Dredge 1398; and 12 = altered glass of Hole 447A; $\mathrm{L}=$ liquid; see text for explanations of Arrows I and II.)

pressure. The initiation of Trend II from approximately the middle of Trend I may indicate that the anchieutectic relations of Trend II had been attained at moderate pressures, between $10 \mathrm{~kb}$ and $1 \mathrm{~atm}$. This case would correspond to crystallization of the melt originating at $10 \mathrm{~kb}$ (Trend I) and cooling of the melt at moderate pressures in an intermediate-level magma chamber. The high $\mathrm{Al}$ content of clinopyroxene phenocrysts from the basalt of Hole 450 (Table 5) seems to confirm this suggestion.

We estimated the formation temperatures of Leg 59 basalts, basing our figures on the well-known geothermometry of olivine-melt equilibrium (Roeder and Emslie, 1970). We obtained preliminary experimental data of phenocryst-melt inclusion thermometry as well (Table 7). As shown in this table, calculated temperatures of olivine-glass equilibria (Roeder and Emslie, 1970) are noticeably lower than homogenization tem-

Table 7. Crystallization temperatures of tholeites of Holes 447A, 449, and 450, DSDP Leg 59.

\begin{tabular}{lc|cc|c}
\hline \multicolumn{1}{c|}{ Hole } & 447A & \multicolumn{2}{|c|}{449} & 450 \\
\hline $\begin{array}{c}\text { Sample } \\
\text { (intervals in cm) }\end{array}$ & $\begin{array}{c}30-5, \\
40-42\end{array}$ & $17-1$, & $17-1$, & $36-3$, \\
\hline $\mathrm{T}^{\circ} \mathrm{C} \mathrm{Ol} / \mathrm{L}^{\mathrm{a}}$ & 1220 & 1210 & 1260 & $32-42$ \\
$\mathrm{~T}^{\circ}$ hom $\mathrm{C}^{\mathrm{b}}$ & - & 1250 & 1250 & 1240 \\
(inclus. in olivine) & - & &
\end{tabular}

${ }^{\text {a }}$ Calculated temperatures of olivine-glass equilibria.

${ }^{b}$ Homogenization temperatures of melt inclusions olivine phenocrysts. 
peratures of melt inclusions of olivine phenocrysts. This may be caused by either of the following:

1) Calculated temperatures of olivine-glass equilibria characterize the temperature of the system immediately before quenching and thus give an estimate of its minimum value. In comparison, homogenization temperatures of melt inclusions from the central parts of olivine phenocrysts represent earlier stages of the crystallization process that are higher in temperature.

2) Olivine-glass equilibrium temperatures were calculated for 1-atm pressure (Roeder and Emslie, 1970), but these temperatures increase with increasing pressure approximately at $5^{\circ} \mathrm{C}$ per $1 \mathrm{~kb}$ (Vaganov and Kuznetsov, 1976). Hence we conclude that the tholeiites we studied crystallized at pressures greater than $1 \mathrm{~atm}$; thus the calculated temperatures were slightly underestimated. The latter is apparently true for Hole $\mathbf{4 5 0}$ tholeiites containing clinopyroxene phenocrysts enriched with alumina. In any case, calculated as well as experimentally obtained temperatures lay in limits established for dry melting conditions.

Basalts of Holes 449, 450, and 54 inherit compositions of respective glasses and are shown as parallel lines in Figure 6. For each of these lines $\mathrm{Ti}, \mathrm{Al}, \mathrm{Ca}, \mathrm{Na}$, and $\mathrm{K}$ increase and $\mathrm{Si}$ and $\mathrm{Mg}$ decrease in the direction from glass to basalt. Total iron displays an irregular pattern along this direction; i.e., it may increase, decrease, or remain constant, although the $\mathrm{FeO}^{*} / \mathrm{MgO}$ ratio distinctly increases toward basalts. Established compositional variation from glasses to rocks cannot be explained by phenocryst fractionation models. This variation may be explained by low-temperature submarine alteration of basalts.

In addition to the basalts of Holes 449,450 , and 54 , submarine alteration processes have considerably affected the upper part of the basement section at Hole 447A (Units 1 and 2). Similar compositional changes have been demonstrated recently by Honnorez et al. (1978) for the submarine alteration process of Atlantic tholeiites. An analogous trend is characteristic for the transition from fresh to altered volcanic glass at Hole 447A (Fig. 6).

Fresh basalts and glasses of the Parece Vela basement reveal a tendency to relative enrichment with $\mathrm{Ti}, \mathrm{Ba}$, and $\mathrm{P}$, compared to those of Hole 447A (Table 6; Figs. $3,5,7$, and 8). Marked enrichment of fresh glasses with potassium (by a factor of approximately 2) is established from Hole 447A to Parece Vela sites. Variation limits of other trace elements, and $\mathrm{Ba} / \mathrm{Sr}, \mathrm{Ce} / \mathrm{Y}$, and $\mathrm{Zr} / \mathrm{Nb}$ ratios, display no marked differences for Parece Vela and West Philippine basin tholeiites, and all are well within the variation limits of normal MORB. Hart et al. (1972) described the nearby Mariana Trough tholeiites (latitude $17^{\circ} \mathrm{N}$ ), which had high titanium content $\left(1.25-1.66 \% \mathrm{TiO}_{2}\right)$ and exhibited a distinct tendency toward enrichment with $\mathrm{K}, \mathrm{Rb}, \mathrm{Sr}, \mathrm{Ba}$, and rare earth elements (REE). Thus the available data allow us to state that along the transect under discussion, tholeiitic basalts of the basement show a slight increase in alkaline tendencies from the western to the eastern basins of the Philippine Sea.

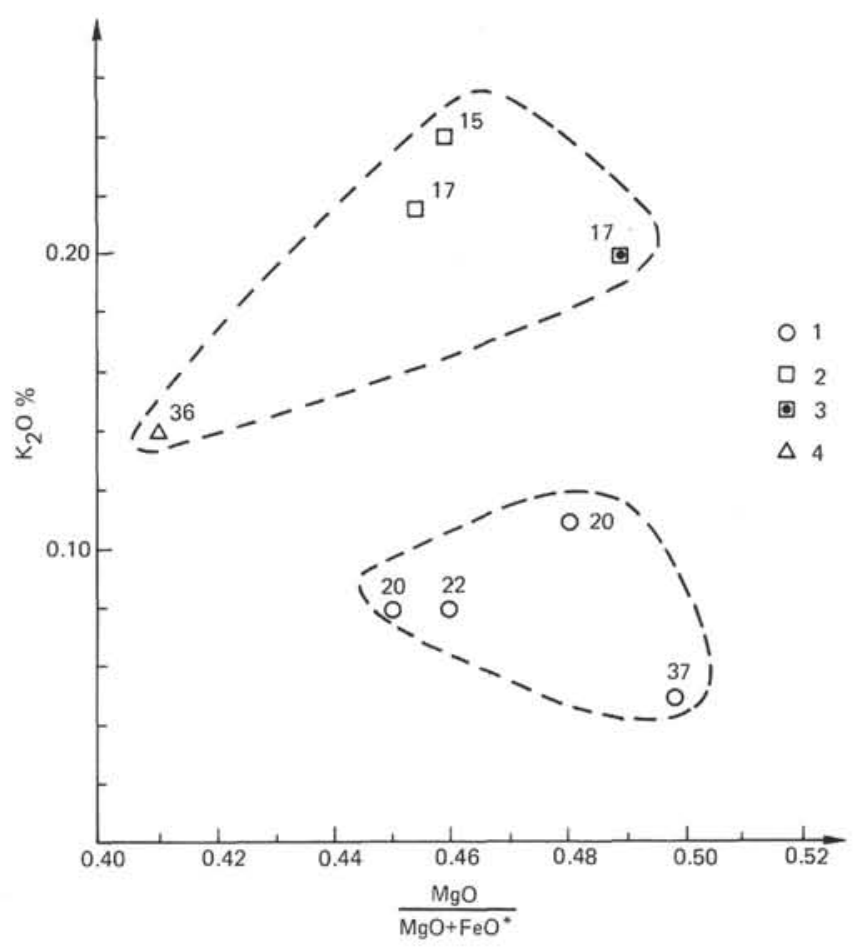

Figure 7. Variation of $\mathrm{K}_{2} \mathrm{O}$ in glasses of Holes $447 \mathrm{~A}, 449$, and 450. (The key is the same as for Fig. 2, except that the square with the dot designates a glass inclusion in an olivine phenocryst [Hole 449].)

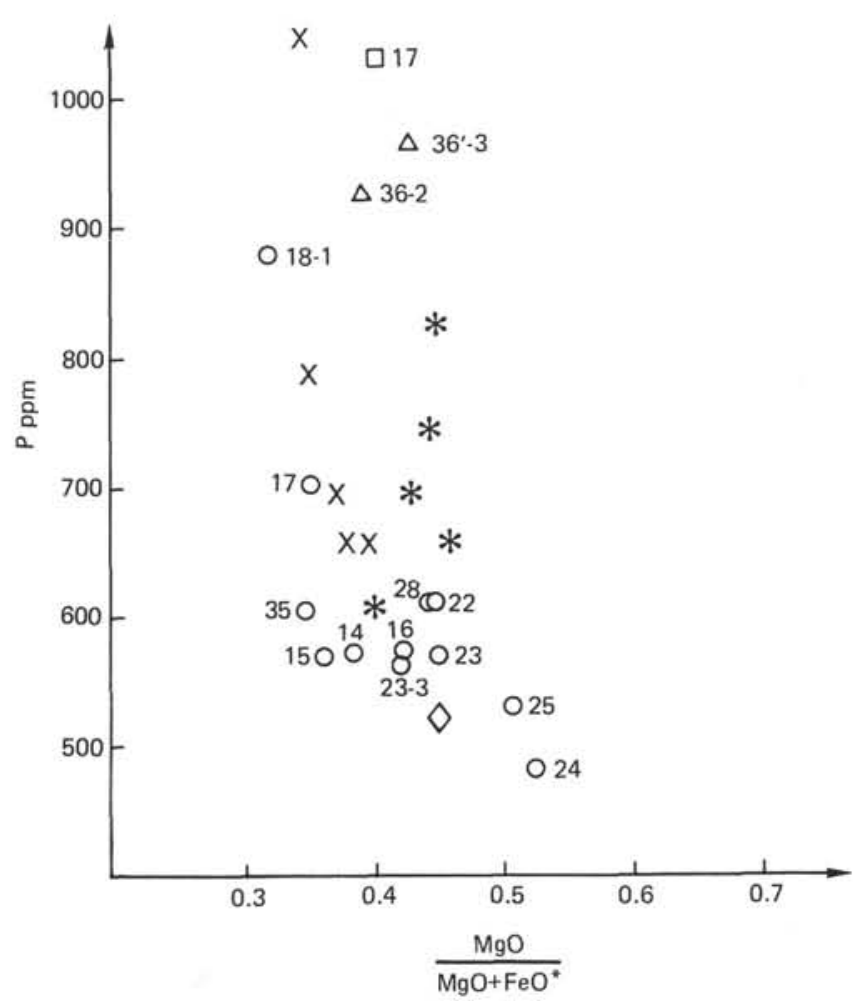

Figure 8. Distribution of phosphorus in West Philippine Basin and Parece Vela Basin tholeiites. (The key is the same as for Fig. 3; asterisks indicate data from Hart et al. [1972].) 


\section{DISCUSSION}

The results of our petrologic investigation of the basement basalts of the West Philippine and Parece Vela basins show that these basalts are essentially indistinguishable from normal (depleted) MOR tholeiites, although the basin basalts are slightly enriched with $\mathrm{Ba}$ (25-43 ppm) and display lower $\mathrm{Zr} / \mathrm{Nb}$ ratios (10.7-22) (Kay et al, 1970; Kay and Hubbard, 1978, Sun et al., in press). The process of evolution of tholeiitic magmatism during formation of Oceanic Layer 2 of such basins occurs under conditions of dry basaltic systems and does not display any petrologic or geochemical evidence of subduction-zone influence (presence of water-bearing phases, enrichment with water and large-ion-lithophyle [LIL] elements, decrease of crystallization temperatures, etc.). In this respect, the geodynamic features of basement formation of these basins in the Philippine Sea plate are, for the most part, very similar to that of mid-ocean ridges.

It is to be emphasized, however, that these petrologic data characterize two different stages of basement formation of the Philippine Sea plate: the West Philippine Basin (50-35 m.y.) and the Parece Vela Basin (30-13 m.y.). It is interesting that according to geologic and geophysical data, the geodynamic evolution of these two basins may not be completely similar. If the Parece Vela Basin is a good example of an extensional back-arc basin, the history of the West Philippine Basin is much more complex (Karig, 1975; Scott et al., this volume). Several alternative models of development of the West Philippine Basin are still disputed: different versions of rapid back-arc spreading; MOR-type spreading followed by entrapment to form marginal basins, and modification of the basement by later intrusion or extrusion from a mosaic of crustal fissures are all postulated models of formation (Karig et al., 1973; Karig, 1975; Louden, 1976; Hilde et al., 1977).

Although the number of basement sections in the present study is rather limited, the petrologic data we obtained do not support the idea of considerable differences in the geodynamic evolution of these two basins. The distinctions between the evolutionary trends of West Philippine and Parece Vela tholeiites are mainly the result of differences in upwelling and differentiation dynamics of primary tholeiitic liquids rather than the result of change in the geodynamic situation. On one hand, the composition of Hole 450 basalts is completely consistent with the differentiation trend of Parece Vela tholeiites and hence apparently reflects the process of basement formation. On the other hand, it is noteworthy that the consecutive stages of basement formation of the Philippine Sea plate (West Philippine Basin, Parece Vela Basin, and Mariana Trough) are characterized by slight but distinct increases of alkaline tendencies of basaltic volcanism. This phenomenon is not explained by the differentiation schemes suggested earlier and possibly reflects the tendency toward increase of depth of the origin of the primary tholeiitic liquids or a decrease in the degree of partial melting of mantle lherzholite. At the first approach, this suggestion seems to be in accord with the tendency of decrease of silica content and increase of normative olivine content of glasses from the West Philippine Basin to the Parece Vela Basin (Green and Ringwood, 1967), although the alumina content does not change considerably. Such a conclusion, however, may be true only for these few samples of the South Philippine Sea transect that we studied. Indeed, the Philippine Sea basalts may display too great a diversity in composition to be consistent with the simple scheme suggested earlier. In particular, Marsh et al. (1978) came to the conclusion that a considerable chemical diversity of mantle sources fed the basalts of Shikoku and Daito basins.

\section{CONCLUSIONS}

1) All the analyzed basement basalts of the basins of the Philippine Sea (Hole 447A-West Philippine Basin -and Holes 449 and 450-Parece Vela Basin) are essentially indistinguishable from MOR-type tholeiites. Volcanism of other types known for large oceanic plates or active continental margins is completely absent in the investigated basement sections.

2) All basalts studied represent differentiation products of primary tholeiitic liquid originating from a dry lherzholitic mantle at temperatures close to $1270^{\circ} \mathrm{C}$ and pressures at about $10 \mathrm{~kb}$.

3) At the earliest stage of fractionation, within pressure limits from 10 to $5 \mathrm{~kb}$, the primary liquid lost a considerable amount of the olivine that was excessive to cotectic crystallization. Further separation of crystallized phases did not take place, and because of this, all these rocks represent the products of cotectic or anchieutectic crystallization at pressures from $5 \mathrm{~kb}$ to 1 atm.

4) The basalts of Hole 447A of the West Philippine Basin are interpreted to be products of cotectic crystallization $(\mathrm{Ol}+\mathrm{Pl})$ of tholeiitic melt at $1 \mathrm{~atm}$, after separation of excessive olivine and rapid uplift of the melt at shallow depth without considerable decrease of temperature. These basalts are represented by a relatively wide range of compositions.

Parece Vela tholeiites represent the products of anchieutectic crystallization $(\mathrm{Cpx}+\mathrm{Pl}+\mathrm{Ol})$ that was initiated at intermediate pressures (between $5 \mathrm{~kb}$ and 1 atm). This may have happened as a result of delay of upwelling magma in the intermediate magma chamber at depths from 3 to $5 \mathrm{~km}$.

5) Basement basalts show a slight increase of alkaline tendencies from the West Philippine Basin to the Parece Vela Basin, and thence to the Mariana Trough. This may reflect a tendency toward increasing depth of primary liquid generation or a decreasing degree of partial melting of the upper mantle source. Such a conclusion, however, may be true only for the South Philippine Sea transect studied on DSDP Leg 59.

\section{REFERENCES}

Bender, J. F., Hodges, F. N., and Bence, A. E., 1978. Petrogenesis of basalts from the project FAMOUS area: experimental study from 0 to 15 k bars. Earth Planet. Sci. Lett., 41:277-302. 
Bougault, H., and Hekinian, R., 1974. Rift valley in Atlantic Ocean near $36^{\circ} 50^{\prime} \mathrm{N}$ : Petrology and geochemistry of basaltic rocks. Earth Planet. Sci. Lett., 24:249-261.

Dietrich, V., Emmerman, R., Keller, G., et al., 1977. Tholeiitic basalts from the Tyrrhenian Sea floor. Earth Planet. Sci. Lett., $36: 285-296$.

Dietrich, V., Emmerman, R., Oberhänsli, H., et al., 1978. Geochemistry of basaltic and gabbroic rocks from the West Mariana Basin and the Mariana Trench. Earth Planet. Sci. Lett., 39:127-144.

Dmitriev, L. V., Sobolev, A. V., and Suschevskaya, N. M., 1978. Primary melt of oceanic tholeiite and composition of upper mantle of ocean. Dokl. Akad. Nauk USSR, 240:177-180. 1979. Conditions of formation of primary melts of oceanic tholeiites and variation of its composition. Geokhimiya, 2:163-178.

Frey, F. A., Bryan, W. B., and Thompson, G., 1974. Atlantic Ocean floor. Geochemistry and petrology of basalts from Legs 2 and 3 of the Deep Sea Drilling Project. J. Geophys. Res., 79:5507-5527.

Fujii, T., and Kushiro, I., 1977. Melting relations and viscosity of an abyssal tholeiite. Carnegie Inst. Washington, Yearb., 76:461-465.

Gill, J. B., 1976. Composition and age of Lau Basin and Ridge volcanic rocks. Implications for evolution of an interarc basin and remnant arc. Bull. Geol. Soc. Am., 37:1384-1395.

Green, D. H., and Ringwood, A. E., 1967. The genesis of basaltic magmas. Contrib. Mineral. Petrol., 15:103-190.

Hart, S. R., Glassley, W. E., and Karig, D. E., 1972. Basalts and sea floor spreading behind the Mariana Island arc. Earth Planet. Sci. Lett., 15:12-18.

Hawkins, J. W., 1976. Petrology and geochemistry of basaltic rocks of the Lau Basin. Earth Planet. Sci. Lett., 28:283-297.

1977. Petrologic and geochemical characteristics of marginal basin basalts. In Talwani, M., and Pitman, W. C., III (Eds.), Island Arcs, Deep Sea Trenches, and Back-Arc Basins: Washington (Am. Geophys. Union), pp. 355-365.

Hilde, T. W. C., Uyeda, S., and Kroenke, L., 1977. Evolution of the Western Pacific and its margin. Tectonophysics, 38: 145-165

Honnorez, J., Bohlke, I. L., and Honnorez, B. M., 1978. Petrological and geochemical study of the low temperature submarine alteration of basalt from Hole 396B, Leg 46. In Dmitriev, L., Heirtzler, J., et al., Init. Repts. DSDP, 46: Washington (U.S. Govt. Printing Office), 299-311.

International Working Group on the IGCP Project "Ophiolites" (Dmitry Mendeleev, June-August, 1976), 1977. Initial reports of the geological study of oceanic crust of the Philippine Sea floor. Ofioliti, 2:137-68.

Karig, D. E., 1971. Origin and development of marginal basins in the Western Pacific. J. Geophys. Res., 76:2542-2561.

1975. Basin genesis in the Philippine Sea. In Karig, D. E., Ingle, J. C., Jr., et al, Init. Repts. DSDP, 31: Washington (U.S. Govt. Printing Office), 857-879.
Karig, D., Ingle, J. C., Bouma, A. H., et al., 1973. Origin of the West Philippine Basin. Nature, 246:458-461.

Kay, R. W., and Hubbard, N. J., 1978. Trace elements in ocean ridge basalts. Earth Planet. Sci. Lett., 38:95-116.

Kay, R., Hubbard, N. J., and Gast, P. W. 1970. Chemical characteristics and origin of oceanic ridge volcanic rocks. J. Geophys. Res., $75: 1585-1613$.

Kushiro, I., 1973. Origin of some magmas in oceanic and circumoceanic regions. Tectonophysics, 17:211-222.

Louden, K. E., 1976. Magnetic anomalies in the West Philippine Basin. In Sutton, G. H., Manghnani, M. H., and Moberly, R. (Eds.), The Geophysics of the Pacific Ocean Basin and Its Margin: Geophys. Monogr. Ser., 19: Washington, D.C. (Am. Geophys. Union), 253-267.

Marsh, N. G., Tarney, J., and Saunders, A. D., 1978. Geochemistry of basalts from the Shikoku and Daito Basins: IPOD Leg 58. Trans. Am. Geophys. Union, 59:1186. (Abstract)

Moberly, R., 1972. Origin of lithosphere behind island arcs, with reference to the Western Pacific. Geol. Soc. Am. Mem., 132: 35-55.

Molnar, P., and Atwater, T., 1978. Interarc spreading and cordilleran tectonics as alternates related to the age of subducted oceanic lithosphere. Earth Planet. Sci. Lett., 41:330-340.

Packham, G. H., and Falvey, D. A., 1971. An hypothesis for the formation of marginal seas in the Western Pacific. Tectonophysics, 11:79-109.

Ridley, W. I., Rhodes, I. M., Reid, A. M., et al., 1974. Basalts from Leg 6 of the Deep Sea Drilling Project. Part 1. J. Petrol., 15:140-159.

Roeder, P. L., and Emslie, R. F., 1970. Olivine-liquid equilibrium. Contrib. Mineral. Petrol., 2:275-289.

Sclater, J. G., Karig, D., Lawver, L. A., et al., 1976. Heat flow, depth, and crustal thickness of the marginal basins of the South Philippine Sea. J. Geophys. Res., 81:309-318.

Sun, S. S., Nesbitt, R., and Sharaskin, A., in press. Geochemical characteristics of mid-ocean ridge basalts. Earth Planet. Sci. Lett.

Tarney, J., Saunders, A. D., and Weaver, S. D., 1977. Geochemistry of volcanic rocks from the island arcs and marginal basins of the Scotia arc region. In Talwani, M., and Pitman, W. C., III (Eds.), Island Arcs, Deep Sea Trenches, and Back-Arc Basins: Washington (Am. Geophys. Union), pp. 367-377.

Toksöz, M. W., and Bird, P., 1977. Formation and evolution of marginal basins and continental plateaus. In Talwani, M., and Pitman, W. C. III (Eds.), Island Arcs, Deep Sea Trenches, and BackArc Basins: Washington (Am. Geophys. Union), pp. 379-393.

Vaganov, V. I., and Kuznetsov, I. E., 1976. Determinations of temperatures of crystallization of olivine for ultrabasic and basic rocks. Modern Methods of Petrologic Investigations: Moscow (Nauka), pp. 142-157. 